\title{
Why different water models predict different structures under 2D confinement
}

\author{
James Dix ${ }^{1}$, Leo Lue ${ }^{2}$, Paola Carbone ${ }^{1, *}$ \\ ${ }^{1}$ School of Chemical Engineering and Analytical Sciences, University of Manchester, M13 9PL, \\ Manchester, UK \\ ${ }^{2}$ Department of Chemical and Process Engineering, University of Strathclyde, James Weir Building, \\ 75 Montrose Street, Glasgow G1 1XJ, United Kingdom
}

Keywords: graphene, confinement, molecular dynamics, water, SPC/E, TIP4P/2005, TIP5P, hydrogen bond

\begin{abstract}
Experiments of nanoconfined water between graphene sheets at high pressure suggest that it forms a square ice structure (G. Algara-Siller, et al. Nature, 519 (2015) 443). Molecular dynamics (MD) simulations have been used to attempt to recreate this structure, but there have been discrepancies in the structure formed by the confined water depending on the simulation set-up that was employed and particularly on the choice of water model. Here, using classical molecular dynamics simulations, we have systematically investigated the effect that three different water models (SPC/E, TIP4P/2005 and TIP5P) have on the structure of water confined between two rigid graphene sheets with a $0.9 \mathrm{~nm}$ separation. We show that the TIP4P/2005 and the TIP5P water models form a hexagonal AA-stacked structure, whereas the SPC/E model forms a rhombic AB-stacked structure. Our work demonstrates that the formation of these structures is driven by differences in the strength of hydrogen bonds predicted by the three water models, and that the nature of the graphene/water interaction only mildly affects the phase diagram. Considering the available experimental data and first-principle simulations we conclude that, among the models tested, the TIP4P/2005 and TIP5P force fields are for now the most reliable when simulating water under confinement.
\end{abstract}




\section{Introduction}

Graphene enclosures with a separation of $\sim 1.0 \mathrm{~nm}$ have been shown to promote chemical reactions ${ }^{[1]}$ and liquid-solid phase transitions ${ }^{[2]}$ that are unexpected in unconfined systems due to the high pressures experienced by the molecules trapped between the two surfaces. ${ }^{[1]}$ The combination of both the high stress and restricted movement in one dimension therefore makes graphene enclosures a distinctive physical environment. To be able to exploit graphene enclosures for uses like chemical reactors, it is important to understand the structure and dynamics of confined molecules under high pressure. These properties can be difficult to obtain from experiments, particularly for water which exhibits a large array of anomalous behavior ${ }^{[3]}$. The recent experiments of Algara-Siller et al. ${ }^{[2]}$ showed that in an enclosure with $\sim 1.0 \mathrm{~nm}$ height, water molecules can form a square ice structure with an AA-stacking conformation (i.e. the water oxygen atoms in each layer lie on top of each other). However, this observation has been debated in the literature ${ }^{[4]}$, highlighting the difficulty of conducting these experiments.

Computer simulation techniques, like molecular dynamics (MD), can aide in the understanding of confined systems that are hard to investigate experimentally. MD has already been shown to be useful in understanding confined systems ${ }^{[1,5-7]}$, however accurately modelling the phase behavior of unconfined water through MD simulations has already proved to be difficult due to its complex phase diagram $^{[8,9]}$. Under confinement, the combination of the limited translational and rotational degrees of freedom and water/surface interactions adds an extra challenge as they can both influence the properties of confined water. Moreover, limited experimental data make it hard to validate the confined models of water and the water/surface interactions.

Despite these difficulties, many studies have used MD simulations to investigate confined water. Previous studies have recreated the high pressure environment by either applying pressure to external reservoirs to increase the water density in the channe ${ }^{[10-12]}$, or by placing a fixed number of water molecules between two infinite parallel sheets to impose a specific density ${ }^{[13-16]}$. These studies have used different water models, including SPC/ $\mathrm{E}^{[16]}, \mathrm{TIP} 4 \mathrm{P} / 2005^{[10-12]}$ and TIP5 $\mathrm{P}^{[14]}$, different interactions between the water molecules and the carbon walls ${ }^{[13,17-20]}$, as well as various sheet-sheet separations, ranging from $0.6-2.0 \mathrm{~nm}^{[10,19]}$. This variety in simulation set ups can predict different confined water 
structures and values of transition pressure ${ }^{[2,10]}$, which makes it difficult to compare between different studies. Moreover most of these simulations use rigid model for the water molecules. Recently, a new type of flexible single-point charge model has been proposed $^{[21]}$. The authors showed that, introducing flexibility into the bonded part of the potential, the model predictions of several liquid state properties such as dielectric constant and viscosity improve. ${ }^{[22]}$ While recent molecular dynamics simulations indicated that adding flexibility into the model does not change the structure of the confined water at ambient conditions ${ }^{[23]}$ this could nevertheless change the liquid/solid transition point. Finally the symmetry of the crystal structure of the confined water could be important in the understanding of the mechanism of electromelting or electrofreezing (i.e. melting or freezing of water induced by the application of an electric field). ${ }^{[24-26]}$ since the water molecules arrangement within the pores influence the phase behaviour of the liquid under electric field.

There is therefore a clear need to test how different water models behave under the same confined environment and to understand what properties of the models control their behavior. In this work, we have aimed at understanding this variation by systematically comparing three common water models (SPC/E, TIP4P/2005 and TIP5P). This was done for a two-dimensional channel comprised of rigid graphene sheets with a separation of $0.9 \mathrm{~nm}$ between the sheets both with and without contact to an external reservoir of water. Although there is limited experimental data, we have observed that only the TIP4P/2005 and TIP5P water models can reproduce an AA-stacked hexagonal structure observed in quantum Monte Carlo calculations ${ }^{[27]}$.

\section{Computational details}

Water and graphene models. In this work we have analysed three common water models which have been used extensively in simulations under confinement ${ }^{[6,10-12,14,17-20]}$ : SPC/ $\mathrm{E}^{[28]}, \mathrm{TIP} 4 \mathrm{P} / 2005^{[29]}$ and TIP5 $\mathrm{P}^{[30]}$. All of these models have a Lennard-Jones 12-6 interaction site at the position of the oxygen, $\mathrm{O}$, atom and positive partial charges on the hydrogen, $\mathrm{H}$, atoms. To counterbalance the partial charges on the hydrogen atoms in SPC/E, a partial negative charge is placed on the $\mathrm{O}$ atom, in the TIP4P/2005 
model it is shifted towards the $\mathrm{H}$ atoms (point $\mathrm{M}$ in Figure 1a), and in the TIP5P model it is split between the two lone pair sites of the oxygen atom (points L in Figure 1a). All of the water models are rigid and non-polarizable (see SI 1 for the values of all parameters). Comparative studies between water models for unconfined water have shown that TIP4P/2005 is the best of these water models at reproducing the experimental phase diagram and densities ${ }^{[8,31,32]}$ of unconfined ice.

a)<smiles>OI</smiles>
SPC/E

c)

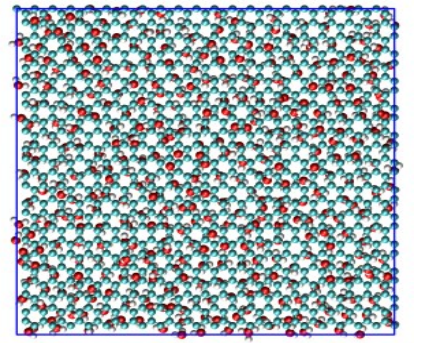

xy - plane

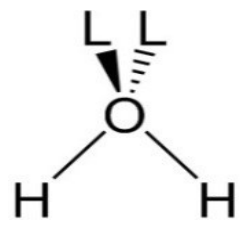

TIP5P

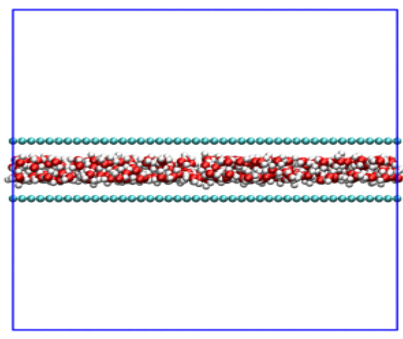

zy - plane b)

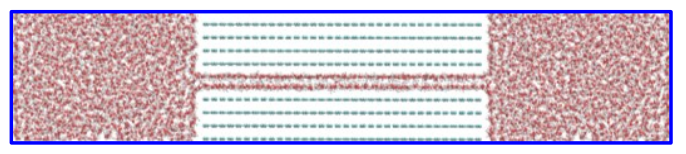

zy - plane

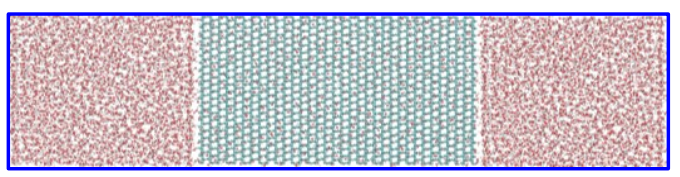

zx - plane

Figure 1. Schematic structures of SPC/E, TIP4P/2005 and TIP5P water models (a), snapshots of for a reservoir simulation (b) and the parallel plate simulation (c).

The water/graphene interaction was modelled by using a Lennard-Jones $12-6$ potential for all of the carbon, $\mathrm{C}$, atoms ${ }^{[19]}$ rather than the 9-3 Lennard Jones "featureless walls" potential ${ }^{[33]}$. This was because the "featureless wall" approach is expected to break down for inter-wall separations below $1.6 \mathrm{~nm}^{[19]}$. Additionally, to capture the structure of water on a surface it is important to use explicit surface atoms as their lattice spacing alters the structure of surface water ${ }^{[34]}$. The parameters for the graphene/water interactions are based on work by Steele et al. ${ }^{[35,36]}$ as they have been used previously to study water confined by graphene sheets or in graphitic slit pores ${ }^{[2,37-40]}$. The C-O cross interaction parameters are shown in Table 1 and are derived from the single atom parameters using the geometric mixing rules 
(see SI 1). To test the sensitivity of the results with the surface/water parameters, simulations with two other graphene force fields $\left(\mathrm{CHARMM}^{[41]}\right.$ and Werder et al. $\left.{ }^{[42]}\right)$ have been carried out (see SI 1).

Table 1. Carbon-oxygen (CO) Lennard-Jones $12-6$ cross term parameters for the SPC/E, the TIP4P/2005 and the TIP5P water models.

\begin{tabular}{c||cc} 
& $\sigma_{C O}(\mathrm{~nm})$ & $\varepsilon_{C O}\left(\mathrm{~kJ} \mathrm{~mol}^{-1}\right)$ \\
\hline \hline SPC/E & 0.328 & 0.388 \\
TIP4P/2005 & 0.328 & 0.424 \\
TIP5P & 0.326 & 0.394
\end{tabular}

Simulation set up. The simulations were performed in two steps: initially a $2 \mathrm{D}$ channel with a $0.9 \mathrm{~nm}$ height and a $7 \mathrm{~nm}$ length, (see SI 2) was connected at either end to an external reservoir, both containing 2000 water molecules (Figure 1b). This channel height was enough to accommodate two layers of water ${ }^{[40]}$. Pressures were applied along the direction of channel (z-axis), $\mathrm{P}_{\mathrm{ZZ}}$, and ranged from 1 bar to 5.0 GPa, but the $\mathrm{x}$ - and $\mathrm{y}$-axis dimensions were kept constant meaning that these simulations were run using the $\mathrm{NP}_{\mathrm{ZZ}} \mathrm{A}_{\mathrm{XY}} \mathrm{T}$ ensemble. These simulations took between 10 and $45 \mathrm{~ns}$ to reach equilibrium (see SI4). The channel density that was reached after the equilibration period was then used to fill another channel between two infinite parallel plates (Figure 1c) (see SI 3), that, due to periodic boundary conditions, acted as an infinite channel and allowed us to remove any influence that the reservoirs and channel entrance had on the water structure. The parallel plate set-up was further run in the NVT ensemble and reached equilibrium within $10 \mathrm{~ns}-80 \mathrm{~ns}$ (see SI 4). The short-range Lennard-Jones, short-range Coulomb and long-range Coulomb interaction energies were monitored to ensure that the systems had reached equilibrium (see SI 5). Comparison between the structure in the parallel-plate and reservoir simulation set-ups showed that both predicted the same structure for the confined water (see SI 6). The reservoir simulations were used to obtain the bond orientational order parameter and channel densities, whereas the parallel plate set-up was used to obtain 2D radial distribution functions, the number of hydrogen bonds and interaction energies (see analysis section). 
All simulations were run using GROMACS 4.5.4 $4^{[43-45]}$. Both simulation set-ups had used the Berendsen thermostat ${ }^{[46]}$ with a coupling constant of $0.2 \mathrm{ps}$ at $300 \mathrm{~K}$ with a $0.5 \mathrm{fs}$ time step with the Leapfrog integrator ${ }^{[47]}$. The short range cut-offs were $1.1 \mathrm{~nm}$ for both the electrostatic and van der Waals interactions. Long-range electrostatic interactions were taken into account using the particle-mesh Ewald summation ${ }^{[48,49]}$ in $3 \mathrm{D}$ and 2D for the reservoir and parallel plate simulations respectively with a grid spacing of $0.12 \mathrm{~nm}$, a fourth order interpolation and a tolerance of $10^{-5}$. The graphene sheets were treated as being neutral and periodic boundary conditions were applied in all three dimensions. Pressure coupling was controlled using the Berendsen barostat ${ }^{[46]}$ with a 4.0 ps coupling constant using a semiisotropic scaling scheme. See SI 2 and 3 for any further details for the simulation set-ups.

Analysis. To quantify the ordering of water molecules between the two confining walls the 2D bond orientational order parameter ${ }^{[50,51]}\left(\psi_{n}\right)$ was calculated. This order parameter produced values between 1 and 0 , with 1 relating to a highly ordered structure and 0 to a disordered structure. The value of $n$ was changed between 4 and 6 , and allowed us to distinguish between the degree of square (or rhombic) and hexagonal ordering respectively, within each layer of confined water. This order parameter was used to analyse the local ordering of water oxygen atoms in each layer separately. Initially a Voronoi tessellation was used to determine all the nearest neighbor oxygen atoms $\left(n_{i}\right)$ for a selected oxygen atom, $i$. For each pair the angle $\left(\theta_{i j}\right)$ between the x-axis, parallel to the sheet, and the vector formed between atom $i$ and its nearest neighbor $j$ was subsequently calculated. These angles were then used in the following equation to calculate $\psi_{n}{ }^{[50,51]}$ :

$\psi_{n}=\frac{1}{N}\left|\sum_{i=1}^{N} \frac{1}{n_{i}} \sum_{j=1}^{n_{i}} \exp \left(i n \theta_{i j}\right)\right|$

where $N$ is the total number of water $\mathrm{O}$ atoms in the channel. These values were calculated using an in-house code that made use of the MDAnalysis Python package ${ }^{[52,53]}$.

The alignment of the two water layers relative to each other was determined from a $2 \mathrm{D}$ radial distribution function $(\mathrm{RDF})^{[14,54]}$. The 2D RDF projects the position of all of the water atoms onto the 
plane of a graphene sheet. Once the transverse position in the channel for all the atoms are removed, one can identify what the relative conformation of atoms between the two layers are. The 2D RDF is given by ${ }^{[5]}$ :

$$
\begin{aligned}
& g_{\|}\left(r_{x y}\right)=\frac{1}{2 \pi \rho z r_{x y} \delta r} \sum_{n=0}^{b} \sum_{i \neq j}\left(H\left(n \delta r-r_{x y}\right)-H\left((n+1) \delta r-r_{x y}\right)\right) \\
& H(x)= \begin{cases}1 & x>0 \\
0 & x \leq 0\end{cases}
\end{aligned}
$$

where $\rho$ is the density of water oxygen atoms, $r_{x y}$ is the distance in the xy-plane of the graphene sheet between atoms $i$ and $j, \mathrm{H}$ is the heavyside function, $\mathrm{b}$ is the number of bins used for the distribution function and $\mathrm{z}$ is the size of the simulation box in the direction perpendicular to the graphene sheets. 


\section{Results}

Confined Water Structure

a)
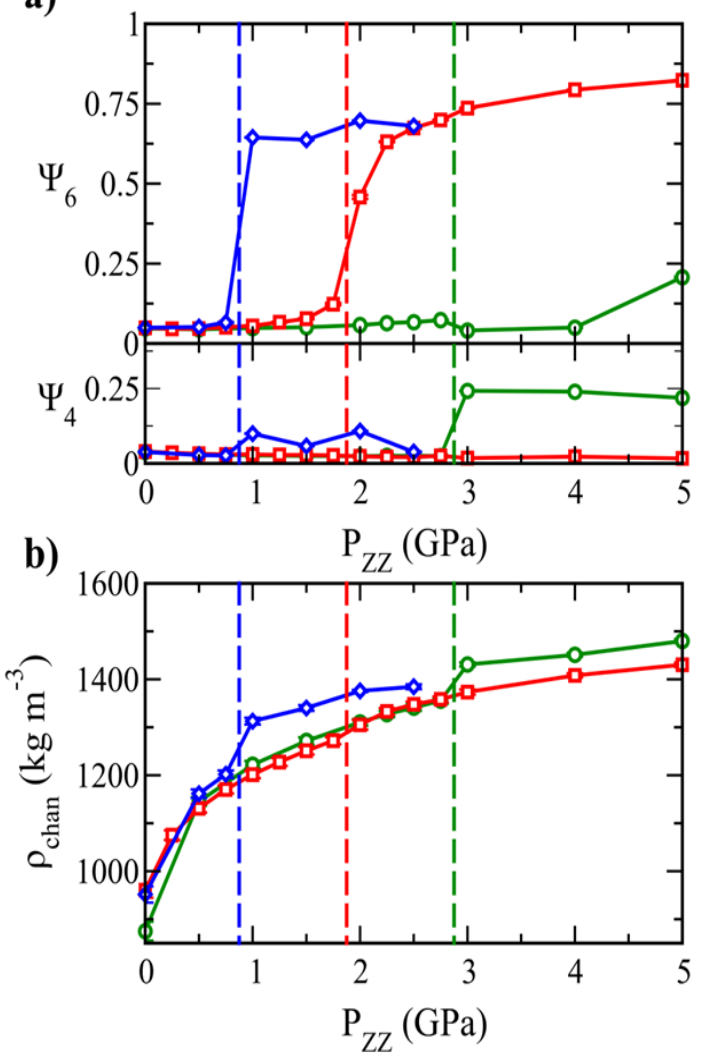

c)
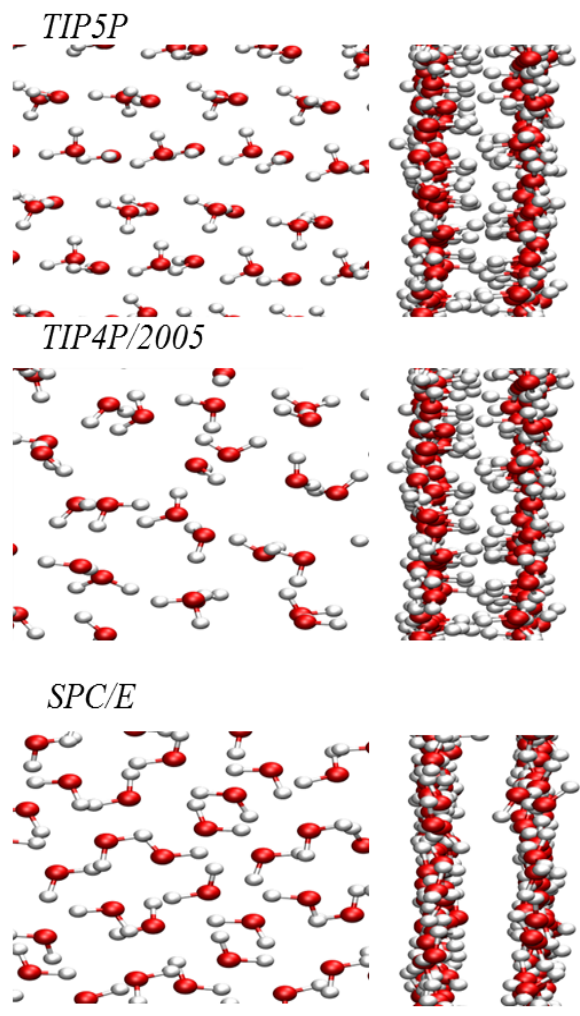

zx-plane

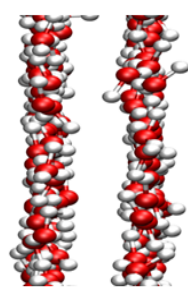

zy-plane

Figure 2. Order parameters, $\psi_{6}$ and $\psi_{4}$ (a) and channel density, $\rho_{\text {chan }}$ (b) plotted against the applied pressure, $\mathrm{P}_{\mathrm{ZZ}}$, for SPC/E (green), TIP4P/2005 (red) and TIP5P (blue). Snapshots of confined water in the zx and zy-planes (c) for TIP5P at 1.0 GPa, TIP4P/2005 at 2.0 GPa and SPC/E at 3.0 GPa. Vertical dashed lines in (a) and (b) correspond to transition points in the order parameter for the water models. See SI 7 for raw data for (a) and (b).

The bond orientational order parameter is a useful tool to follow the changes in the structure of water upon changing the channel pressure and is an effective way to highlight the differences between the water models. We observe that for both TIP4P/2005 and TIP5P, as the applied pressure increases the hexagonal component $(\mathrm{n}=6)$ of the bond orientational order parameter, $\psi_{6}$, also increases (Figure 2a). The emergence of hexagonal symmetry occurs however at different pressures for the two models. TIP5P 
shows hexagonal symmetry at $1 \mathrm{GPa}$ while TIP4P/2005 needs to reach $2 \mathrm{GPa}$. Neither model showed any presence of square symmetry associated to the value of $\psi_{4}$. SPC/E requires a higher pressure of 3 GPa to achieve an ordered structure (Figure 2a) which shows, contrary to the other two models, a square or rhombic symmetry $\left(\psi_{4} \approx 0.25\right)$ and no hexagonal symmetry $\left(\psi_{6} \approx 0.05\right)$. Here it is important to notice that a value of $\psi_{4} \approx 0.25$ is too low for a perfect square structure and is more indicative of a rhombic structure.

The pressure values at which the order parameters $\psi_{4}$ and $\psi_{6}$ change are marked on Figure 2a by dashed vertical lines. These lines coincide with the position of discontinuities in the water channel density, $\rho_{\text {chan }}$ (Figure $2 \mathrm{~b}$ ). At 1 bar pressure SPC/E is the model that predicts the lowest channel density of $860 \mathrm{~kg} \mathrm{~m}^{-3}$, which is substantially smaller than the bulk density value of $996.5 \mathrm{~kg} \mathrm{~m}^{-3}$ at 1 bar and $300 \mathrm{~K}^{[55]}$. The other two water models predict a density more similar to that of bulk water of $960 \mathrm{~kg} \mathrm{~m}^{-}$ ${ }^{3}$ and $930 \mathrm{~kg} \mathrm{~m}^{-3}$ for TIP4P/2005 and TIP5P respectively (Figure 2b). As the pressure increases these initial differences in density between the water models fade away.

A top-down view through the graphene sheets (Figure 2c) show that TIP4P/2005 and TIP5P form a hexagonal structure where the oxygen atoms in the two layers sit on-top of each other. The hexagonal structure gives rise to large values of $\psi_{6}$ in Figure 2a. A snapshot for SPC/E (Figure 2c) highlights, instead, its rhombic structure and that the oxygen atoms in each layer are offset. Side-on snapshots of the water molecules in the graphene channel shows that TIP4P/2005 and TIP5P have hydrogen atoms pointing between the two water layers, whereas SPC/E has no hydrogen atoms connecting the two layers. 


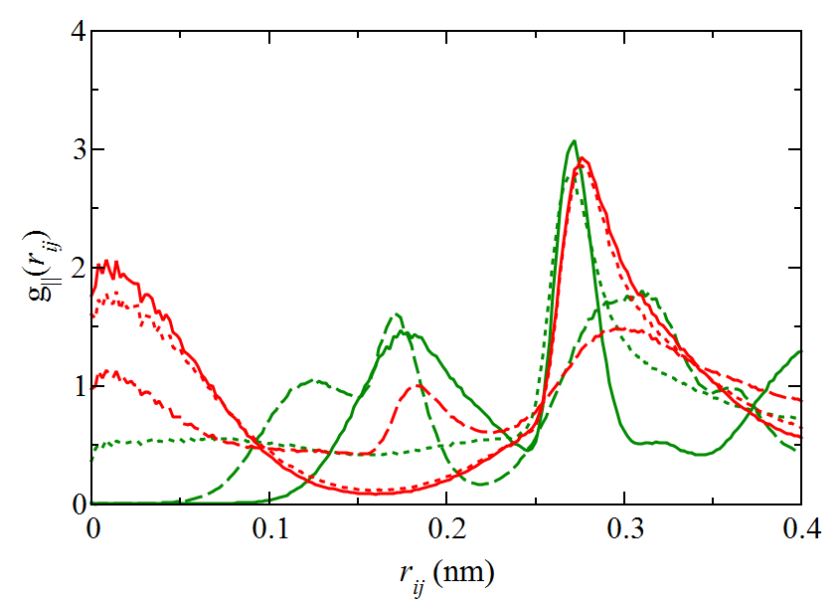

Figure 3. 2D oxygen-oxygen radial distribution function $\left(g_{\|}\left(r_{i j}\right)\right)$ for SPC/E calculated at $2.75 \mathrm{GPa}$ (green, dotted) and 3.0 GPa (green, solid), TIP4P/2005 at $1.75 \mathrm{GPa}$ (red, dotted) and $2.0 \mathrm{GPa}$ (red, solid), and 2D oxygen-hydrogen radial distribution function for SPC/E at 3.0 GPa (green, dashed) and TIP4P/2005 at $2.0 \mathrm{GPa}$ (red, dashed).

A more quantitative analysis of the stacking geometry between the two layers of water can be achieved by looking at the 2D RDF. The 2D RDF looks at the projection of the positions of the atoms onto a graphene sheet. This means that the vertical distance between atoms is ignored and allows for peaks at separations less than the first peak in a 3D O-O RDF ${ }^{[28]}$. In this case any peak below the O-O separation of $0.28 \mathrm{~nm}^{[28,29]}$ is due to the two atoms being in different layers. For the ordered TIP4P/2005 structure the position of the first peak in the oxygen-oxygen RDF, calculated at $2.0 \mathrm{GPa}$, lies close to $r_{i j}=0$ (Figure 3). This indicates that the oxygen atoms in the two layers sit directly on top of each other, in what is called an AA-stacking configuration ${ }^{[2]}$, which agrees with the snapshot in Figure 2c. On the contrary, the ordered SPC/E structure presents a first peak of the RDF around $0.176 \mathrm{~nm}$, which indicates that the oxygen atoms in the two layers are offset by this amount in an AB-stacking configuration. Additionally, the oxygen-hydrogen 2D RDF for the ordered SPC/E structure presents a peak in a similar position to the oxygen-oxygen $2 \mathrm{D}$ RDF. This indicates that in this case the hydrogen atoms sit above the oxygen $\mathrm{O}$ atoms of the layer below (Figure 3). 
It is interesting to notice that TIP4P/2005 already shows evidence of AA-stacking before it undergoes the ordering transition as in the 2D RDF calculated at $1.75 \mathrm{GPa}$ (Figure 3) peak around $r_{i j}=0$ can be already observed. On the contrary, the SPC/E model does not show any ordering until it forms a completely ordered structure.

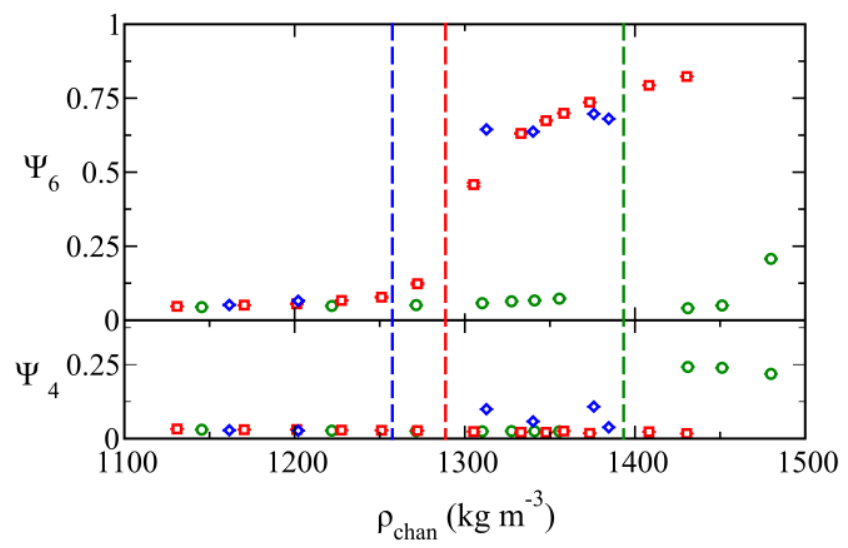

Figure 4. Orientational bond order parameters, $\psi_{6}$ and $\psi_{4}$, plotted against the channel water density, $\rho_{\text {chan }}$, for SPC/E (green), TIP4P/2005 (red) and TIP5P (blue). Dashed vertical lines correspond to the transition points in the order parameter for the water models. See SI 7 for raw data.

Figure 2a seems to indicate that the TIP4P/2005 and TIP5P models form hexagonal structures at different pressures, however when the values of $\psi_{6}$ are plotted against the channel water density, $\rho_{\text {chan }}$ , (Figure 4), it appears clear that both models form a hexagonal structure in a similar density range (1272 - $1305 \mathrm{~kg} \mathrm{~m}^{-3}$ for TIP4P/2005 and $1202-1313 \mathrm{~kg} \mathrm{~m}^{-3}$ for TIP5P). SPC/E requires a instead higher density (between $1356-1431 \mathrm{~kg} \mathrm{~m}^{-3}$ ) to form an ordered rhombic structure (Figure 4). 

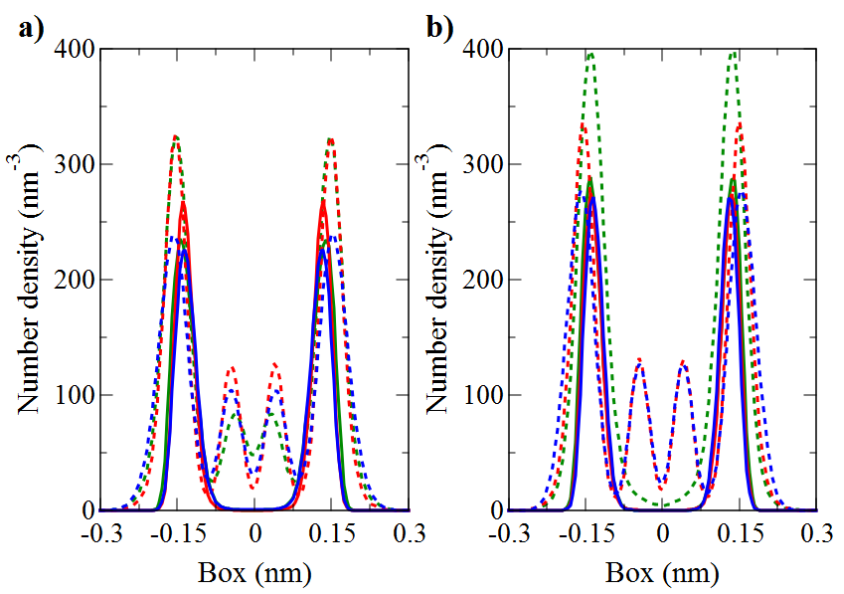

Figure 5. Oxygen (solid lines) and hydrogen (dotted lines) number density profiles for SPC/E (green), TIP4P/2005 (red) and TIP5P (blue) calculated both before (a) and after (b) they have undergone an ordering transition. The pressures for SPC/E are $2.75 \mathrm{GPa}$ (a) and $3.0 \mathrm{GPa}(\mathrm{b}), \mathrm{TIP} 4 \mathrm{P} / 2005$ are 1.75 $\mathrm{GPa}(\mathrm{a})$ and $2.0 \mathrm{GPa}(\mathrm{b})$ and for TIP5P are $0.75 \mathrm{GPa}(\mathrm{a})$ and $1.0 \mathrm{GPa}(\mathrm{b})$.

From the mass density profile calculated across the capillary (Figure 5), it appears clear that for all three water models the oxygen atoms form two separate layers and show a similar position in channel both before (Figure 5a) and after (Figure 5b) the ordering transition takes place. After going through the ordering transition the two layers of oxygen atoms are slightly closer to each other by $0.01 \mathrm{~nm}$ for SPC/E and TIP4P/2005 and $0.005 \mathrm{~nm}$ for TIP5P. Hydrogen atoms arrange into two positions: inbetween the two planes formed by the oxygen atoms or in the same plane as the oxygen atoms (Figure 5). Before ordering, all three water models have hydrogen atom peaks in both positions. After ordering, TIP4P/2005 and TIP5P still show peaks in both positions, while for SPC/E the hydrogen atoms are mainly in the same planes as the oxygen atoms (Figure $5 \mathrm{~b}$ ). This suggests that there are very few hydrogen bonds between the two water layers for the SPC/E model, which agrees with the snapshots in Figure 2c.

Previous simulation work has observed the AB-stacked rhombic SPC/E structure ${ }^{[2]}$. The AA-stacked hexagonal structure has been observed for TIP4P/2005 $5^{[10]}$, as well as for TIP5P confined between featureless graphene walls ${ }^{[14]}$ and silica sheets ${ }^{[56]}$. The latter finding suggests that the structure of confined water is not very sensitive to the graphene/water interaction potential. To test this further we 
have investigated different commonly used carbon-oxygen interaction parameters using the LennardJones 12-6 potential. We observed a slight variation in the transition density and pressure but no noticeable effects on the confined water structure (see SI 1\&9). These results lead us to conclude that the water model has the most dominant role in determining the ordered structure of confined water.

Of particular importance is the water model's capability to form hydrogen bonds (HBs). To test the relative importance of HB formation (compared with the van der Waals attraction) on the geometry of the predicted crystal structures, we performed a set of simulations without electrostatic potential. When the electrostatic interactions, and by proxy hydrogen bonding, are turned off, all the three water models present a new structure with an AB-stacked hexagonal symmetry (see SI 10). This suggests that the atom charge distributions and the structure and strength of hydrogen bonds control the differences in the structures for the three water models.

This is not surprising as the HB network geometry also determines the symmetry and relative stability of the various ice structures found in $3 \mathrm{D}$ ice ${ }^{[57]}$. In particular, Vega and coworkers ${ }^{[8,32,58]}$ have recently shown that water models with a relative strength between dipolar $(\mu)$ and quadrupolar $\left(Q_{T}\right)$ forces closer to the experimental value of 0.9 (in the gas phase ${ }^{[58]}$ ) are those that yield qualitatively better phase diagrams. The authors showed that the key factor that determines the model quality is the position of the negative charge and that models whose ratio of the dipole to quadrupole moment is similar produce, similar phase diagrams. They also noticed that those models that fail to possess a reasonable value for the dipole and quadrupole moments ratio overestimate the stability of the ice II structure (with rhombohedral symmetry), which becomes the most stable ice structure at the expense of ice III and ice V. As a consequence of these observations, models such as SPC/E and TIP5P with $\mu / Q_{T}$ much larger than 1 poorly predict the structure of the solid phases, whereas the TIP4P/2005 model, characterized by a $\mu / Q_{T}$ close to 1 , yield to a qualitatively correct phase diagram. In the present case, the value of the dipole moment or of the $\mu / Q_{T}$ ratio does not seem to correlate with the structures the models predict. TIP4P/2005 and TIP5P are characterized by different $\mu$ and $\mu / Q_{T}$ values ${ }^{[8]}$ still they predict similar structures (hexagonal, AA stacking) with similar transition densities. A similar observation can be made 
when looking at the predicted densities for the unconfined solid phases, where again a clear trend between models cannot be found. TIP4P/2005 is the model that best predicts the densities of all the ice structures (within 1 per cent error), whereas TIP5P is the model characterized by the worst predicted densities (with an overestimation of around 7\%) ${ }^{25}$. Despite this, these models show similar transition densities under confinement. It is therefore possible that under extreme geometrical confinement, when the spatial rearrangement of the molecules is limited, the bond geometry (e.g. the $\mathrm{OH}$ bond length and the $\mathrm{HOH}$ bond angle) is the most defining feature of the system. The TIP5P and TIP4P/2005 models have indeed the same bonded parameters.

\section{Hydrogen bonds}

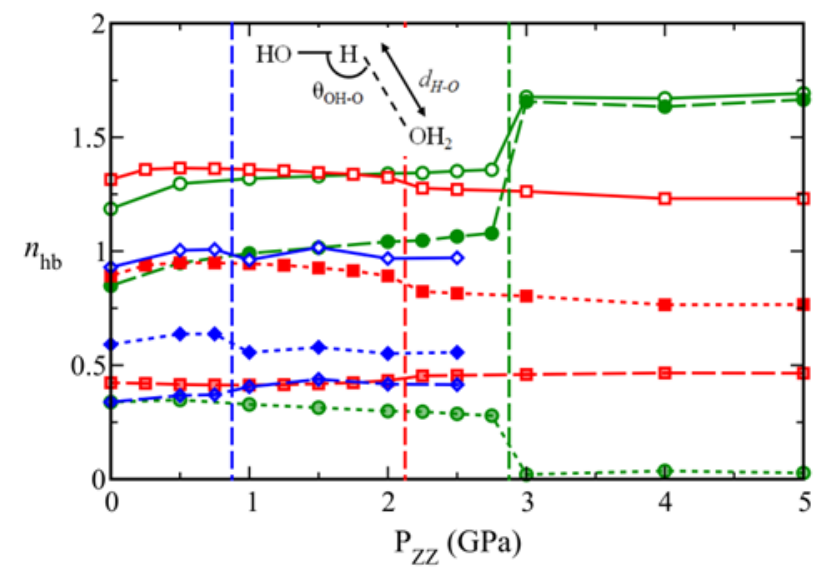

Figure 6. Average number of hydrogen bonds per water molecule, $n_{h b}$, against the applied pressure, $\mathrm{P}_{\mathrm{zz}}$, calculated for SPC/E (green), TIP4P/2005 (red) and TIP5P (blue). Total number of hydrogen bonds - solid lines, hydrogen bonds within the plane - dashed lines, hydrogen bonds between water layers dotted lines. Dashed vertical lines correspond to the transition pressure for the water models. The scheme shows the definition of $\theta_{\mathrm{OH}-\mathrm{O}}$ and $d_{\mathrm{H}-\mathrm{O}}$ used to determine the presence of a hydrogen bond.

To understand the underlying reason behind the formation of the different structures, we calculated the average number of hydrogen bonds per water molecule $\left(n_{h b}\right)$. Several different methods have been proposed to define a hydrogen bond, based typically on either energetic or geometric criteria. ${ }^{[59]} \mathrm{We}$ have opted for geometric criteria. The schematic in Figure 6 shows the definition we used to identify a 
hydrogen bond which involves the O-H---O angle $\left(\theta_{\mathrm{OH}-\mathrm{O}}\right)$ formed between the oxygen and hydrogen atoms of the donor water molecule and the oxygen atom of the acceptor water molecule, and the distance between the hydrogen atom of the donor molecule and the oxygen atom of the acceptor molecule ( $d_{H-O}$ ). In our analysis we identified a hydrogen bond when $\theta_{O H-O}>130^{\circ}$ and $d_{H-O}<0.24 \mathrm{~nm}$. These criteria are similar to those used in other simulations with water ${ }^{[60-62]}$ and were verified using $2 \mathrm{D}$ contour plots (see SI 11), which is a common method to determine the geometric criteria for dilute ${ }^{[63]}$ and condensed ${ }^{[64]}$ polymer systems. As there are two layers of water within the channel, an additional criterion was used to differentiate between hydrogen bonds that are within a layer (intralayer) and between the layers (interlayer). When the difference in the z-coordinate between the donor and acceptor atoms is greater than half the length of the hydrogen bond then it is classed as being between the two layers. The analysis shows that when in the disordered state, TIP4P/2005 and SPC/E possess a higher number of hydrogen bonds per molecule compared to TIP5P (Figure 6). Additionally both TIP4P/2005 and TIP5P prefer to form interlayer hydrogen bonds, whereas SPC/E mainly forms intralayer hydrogen bonds (Figure 6). Once SPC/E goes through the ordering transition, the number of interlayer hydrogen bonds reduces dramatically, mainly leaving intralayer hydrogen bonds in the ordered structure. This is contrary to what is observed for TIP4P/2005 and TIP5P, where interlayer hydrogen bonds still dominate over intralayer ones.

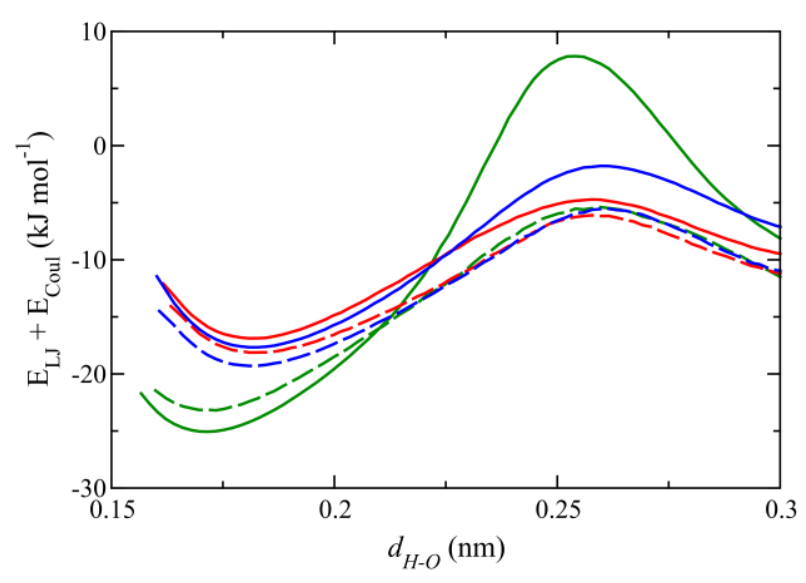


Figure 7. Sum of the short range Lennard-Jones and Coulomb interaction energies for pairs of water molecules against the distance between the hydrogen and the oxygen atoms forming a hydrogen bond, $d_{H-O}$ calculated for SPC/E (green) at 1 bar (dashed) and $3.0 \mathrm{GPa}$ (solid), TIP4P/2005 (red) at 1 bar (dashed) and 2.0 GPa (solid), TIP5P (blue) at 1bar (dashed) and 1.0 GPa (solid).

Analysis of the energetics of forming a hydrogen bond can help to clarify the cause of the different confined structure for the water models. The main energetic trade off to form a hydrogen bond is between the increasingly attractive electrostatic energy against the increasingly repulsive energy of the Lennard-Jones potential. As the Lennard-Jones energy varies as $r^{-12}$ whereas the Coulomb energy varies as $r^{-1}$, at small separations the Lennard-Jones energetic term will dominate over the electrostatic one. At larger separations the reverse is true, meaning that there are energy minima at short separations between the water molecules where hydrogen bonds are formed. These minima can be easily identified as the global minima for all three water models reported in Figure 7.

As found for the liquid state ${ }^{[65]}, \mathrm{SPC} / \mathrm{E}$ has both the strongest and shortest hydrogen bonds of all the three water models (Figure 7), whereas TIP4P/2005 and TIP5P present similar hydrogen bond strengths and lengths, which suggests that they would show similar interactions and form similar structures, as we have indeed observed. 

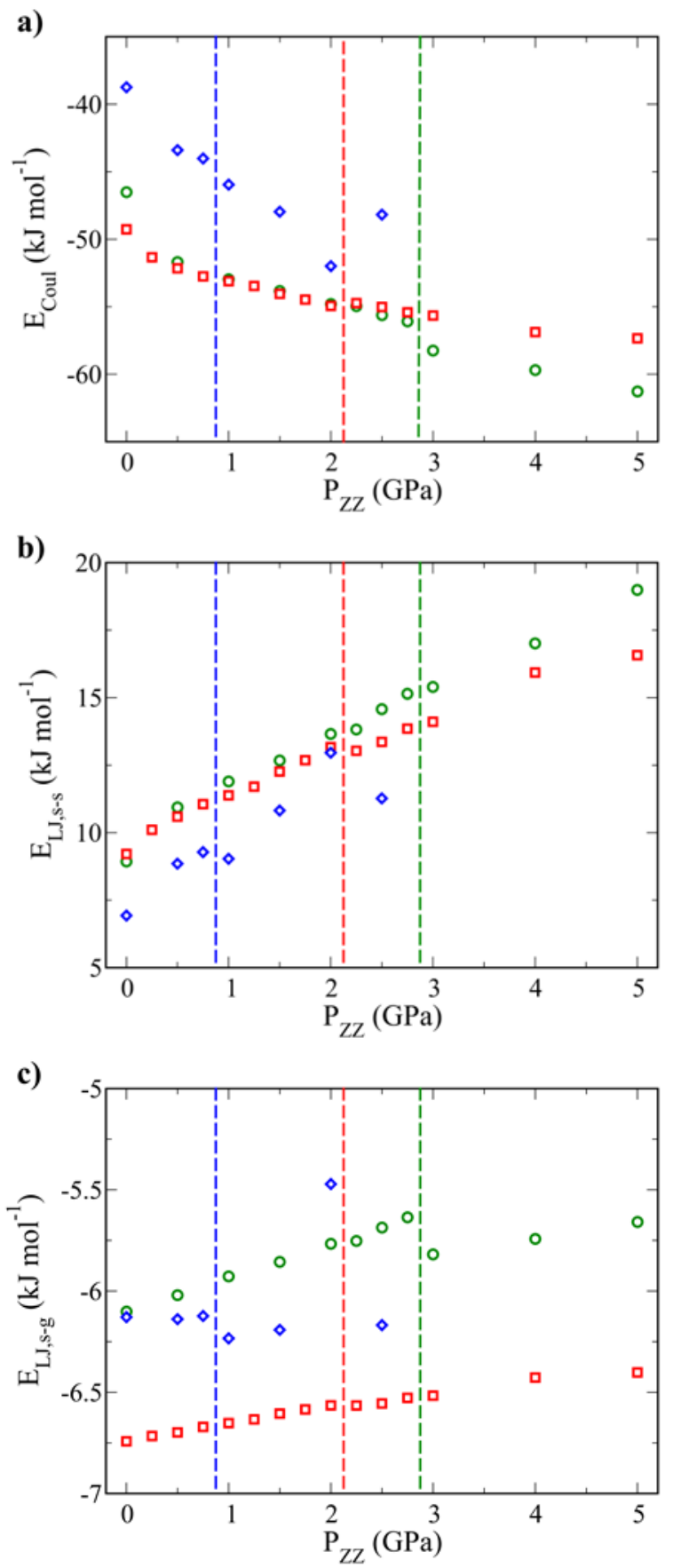

Figure 8. Short-range Coulomb interaction energy (a), solvent-solvent Lennard-Jones interaction energy (b), solvent-graphene Lennard-Jones interaction energy (c) all per water molecule against applied pressure, $\mathrm{P}_{\mathrm{ZZ}}$, for SPC/E (green), TIP4P/2005 (red) and TIP5P (blue). Dashed vertical lines show transition pressure.

For the SPC/E model, as the pressure increases the strength of a single hydrogen bond increases as well

(Figure 7) and as a consequence at the transition pressure the number of hydrogen bonds increases 
(Figure 6). However for the other two models, increasing the pressure and forcing the system to order lead to weaker hydrogen bonds and upon ordering the number of hydrogen bonds overall remain constant.

Looking more in details in the various energetic components we notice that, as expected, for all the water models, as the pressure increases the Coulomb energy becomes more negative (Figure 8a) while the Lennard-Jones energy becomes more positive (Figure 8b). TIP4P/2005 and SPC/E have similar values for both the Coulomb and Lennard-Jones interaction energies (in agreement with their similar water channel densities (Figure 2b) and number of hydrogen bonds (Figure 6)). TIP5P instead predicts a smaller Coulomb and Lennard-Jones interaction energies compared to the other two water models (and indeed presents the smaller number of hydrogen bonds). It is also interesting noticing that the TIP4P/2005 model presents the most attractive solvent-graphene interaction energy, while SPC/E has the least attractive interaction energy (Figure 8c and Table 1), in agreement with the position of the oxygen atoms within the capillary shown in Figure 5.

The energy plots of Figure 8 clarify the reason behind the different structures predicted by the three water models analysed here. For the SPC/E model, the minimization of the potential energy under high pressures (Figures 8a and $\mathrm{b}$ ) is driven by the formation of strong water-water hydrogen bonds which, being fairly shorter than in the other two models (Figure 7), tend to be formed predominantly in plane (i.e. within the same layer) and with a rhombic symmetry to enable each water molecule to both easily accept and donate 2 hydrogen bonds. The limited number of interlayer hydrogen bonds drives a final structure characterized by an AB-stacked geometry that minimizes the Lennard-Jones repulsion. On the contrary the TIP4P/2005 and TIP5P models form significantly weaker hydrogen bonds that upon increase in pressure do not substantially contribute in stabilizing the ordered structure. Indeed at the transition pressure, the number of hydrogen bonds overall slightly decreases for the TIP4P/2005 (figure 6) for which it can also be observed a small increase in the electrostatic interactions (Figure 7a), while stays almost constant for the TIP5P model (Figure 6). To reduce the Lennard-Jones interaction, the oxygen atoms of both models crystallize with a hexagonal symmetry within each layer (i.e. the structures that minimizes the Lennard-Jones interactions, see SI 10) (Figure 2a). The hexagonal 
structure hinders the formation of only intralayer hydrogen bonds and promotes a combination of both inter and intralayer hydrogen bonds. The hydrogen bonding between the layers can overcome some of the Lennard-Jones repulsion causing both TIP4P/2005 and TIP5P to form an ordered AA-stacked structure.

\section{Conclusions}

The aim of this work is to provide a systematic comparison between the structures and transition pressures predicted under nanoscopic confinement by three of the most common classical water models. Our study shows that the three models predict different structures and different transition pressures, although the 4-site and 5-site models show a very similar behavior in terms of structure (hexagonal), layer arrangement (AA-stacked) and transition density value. The SPC/E instead forms a rhombic ABstacked structure and requires much higher pressure (density) to order. These results seem to not directly correlate with the models dipole or quadrupole moments (or their ratio), but with the models bonded parameters and hydrogen bonds network geometry. Based on a detailed analysis of the hydrogen bond number and energetics, it appears clear that SPC/E predicts the formation of stronger and shorter hydrogen bonds compared to the other two models that favor in-plane hydrogen bonds and force the oxygen atoms of the two layer off-set. The other two water models show weaker and longer hydrogen bonds, leading to structures with hexagonal symmetry and allowing for AA-stacking of the oxygen atoms. Finally our results show that the formation of the different ordered structures at high pressure is driven by the water-water interactions rather than by the water/surface ones, indicating that similar phase behavior should be observed in different confinement systems.

Due to the current lack of robust experimental data, it is difficult to determine which model is the best suited for simulations under confinement. Therefore, care should be taken when analysing the results of confined simulations. Recent density functional theory calculations at $0 \mathrm{~K}$ have suggested that for the interlayer distance of $0.9 \mathrm{~nm}$, investigated here, and for pressures above $0.5 \mathrm{GPa}$, a hexagonally close packed structure is the most stable structure ${ }^{[27]}$. Although this work did not include the investigation of $\mathrm{AB}$-stacked structures, it could suggest that the TIP4P/2005 and the TIP5P models are 
more accurate than the SPC/E model. As the experimental techniques to investigate nanoconfined systems are constantly improving, future experiments like Raman or dielectric spectroscopy, could help to verify which model is the most accurate at reproducing the confined structure of water.

\section{Supplementary Material}

See the supplementary material for; interaction parameters (SI 1), reservoir simulation set-up (SI 2), parallel plate simulation set-up (SI 3), simulation equilibration times (SI 4), analysis for simulations reaching equilibrium (SI 5), comparison between parallel plate and reservoir simulation set-up (SI 6), raw data for bond orientational order parameters and channel densities (SI 7), channel density for different interaction parameters (SI 8), confined water structure for different interaction parameters (SI 9), simulation results when electrostatic interaction are removed (SI 10) and 2D contour plots for $\theta_{\mathrm{OH}-\mathrm{O}}$ against $d_{H-O}$ (SI 11).

\section{Acknowledgements}

This work was supported by the North-West Nanoscience Doctoral Training Centre, EPSRC grant EP/G03737X/1. The authors would also like to acknowledge the assistance given by IT Services and the use of the Computational Shared Facility at The University of Manchester. The authors would also like to thank Dr Carlos Avendaño for his guidance on particle ordering.

\section{References}

[1] K. S. Vasu, E. Prestat, J. Abraham, J. Dix, R. J. Kashtiban, J. Beheshtian, J. Sloan, P. Carbone, M. Neek-Amal, S. J. Haigh, et al., Nat. Commun. 2016, 7, 12168.

[2] G. Algara-Siller, O. Lehtinen, F. C. Wang, R. R. Nair, U. Kaiser, H. A. Wu, A. K. Geim, I. V Grigorieva, Nature 2015, 519, 443-445.

[3] P. Gallo, K. Amann-Winkel, C. A. Angell, M. A. Anisimov, F. Caupin, C. Chakravarty, E. Lascaris, T. Loerting, A. Z. Panagiotopoulos, J. Russo, et al., Chem. Rev. 2016, 116, $7463-$ 7500 .

[4] W. Zhou, K. Yin, C. Wang, Y. Zhang, T. Xu, A. Borisevich, L. Sun, J. C. Idrobo, M. F. 
Chisholm, S. T. Pantelides, et al., Nature 2015, 528, E1-E2.

[5] R. R. Nair, H. A. Wu, P. N. Jayaram, I. V. Grigorieva, A. K. Geim, Science 2012, 335, 442444.

[6] R. K. Joshi, P. Carbone, F. C. Wang, V. G. Kravets, Y. Su, I. V. Grigorieva, H. A. Wu, A. K. Geim, R. R. Nair, Science (80-. ). 2014, 343, 752-754.

[7] V. Satarifard, M. Mousaei, F. Hadadi, J. Dix, M. Sobrino Fernandez, P. Carbone, J. Beheshtian, F. M. Peeters, M. Neek-Amal, Phys. Rev. B 2017, 95, 1-8.

[8] J. L. F. Abascal, C. Vega, J. Phys. Chem. C 2007, 111, 15811-15822.

[9] C. Vega, E. Sanz, J. L. F. Abascal, E. G. Noya, J. Phys. Condens. Matter 2008, 20, 153101.

[10] Y. B. Zhu, F. C. Wang, J. Bai, X. C. Zeng, H. A. Wu, ACS Nano 2015, 9, 12197-12204.

[11] Y. Zhu, F. Wang, J. Bai, X. C. Zeng, H. Wu, Phys. Chem. Chem. Phys. 2016, 18, 2203922046.

[12] Y. B. Zhu, F. C. Wang, J. Bai, X. C. Zeng, H. A. Wu, J. Phys. Chem. C 2016, 120, 8109-8115.

[13] K. Koga, X. Zeng, H. Tanaka, Phys. Rev. Lett. 1997, 79, 5262-5265.

[14] J. Bai, X. C. Zeng, Proc. Natl. Acad. Sci. U. S. A. 2012, 109, 21240-5.

[15] K. Koga, H. Tanaka, J. Chem. Phys. 2005, 122, 104711.

[16] N. Giovambattista, P. J. Rossky, P. G. Debenedetti, Phys. Rev. Lett. 2009, 102, 6-9.

[17] A. Striolo, P. K. Naicker, A. A. Chialvo, P. T. Cummings, K. E. Gubbins, Adsorption 2005, $11,397-401$.

[18] S. A. Deshmukh, G. Kamath, S. K. R. S. Sankaranarayanan, Soft Matter 2014, 10, 4067-4083.

[19] A. Striolo, A. A. Chialvo, P. T. Cummings, K. E. Gubbins, Langmuir 2003, 19, 8583-8591.

[20] S. Deshmukh, G. Kamath, S. K. R. S. Sankaranarayanan, ChemPhysChem 2014, 15, 1632 1642.

[21] Y. Wu, H. L. Tepper, G. A. Voth, J. Chem. Phys. 2006, 124, 24503.

[22] G. Raabe, R. J. Sadus, J. Chem. Phys. 2011, 134, 234501.

[23] G. Brekke-Svaland, F. Bresme, J. Phys. Chem. C 2018, 122, 7321-7330.

[24] H. Qiu, W. Guo, Phys. Rev. Lett. 2013, 110, 195701.

[25] R. Zangi, A. E. Mark, Phys. Rev. Lett. 2003, 91, 25502.

[26] A. Bose, A. K. Metya, J. K. Singh, Phys. Chem. Chem. Phys. 2015, 17, 23147-23154.

[27] J. Chen, G. Schusteritsch, C. J. Pickard, C. G. Salzmann, A. Michaelides, Phys. Rev. B 2017, 95, 94121.

[28] H. J. C. Berendsen, J. R. Grigera, T. P. Straatsma, J. Phys. Chem. 1987, 91, 6269-6271.

[29] J. L. Abascal, C. Vega, J. Chem. Phys. 2005, 123, 234505.

[30] M. W. Mahoney, W. L. Jorgensen, J. Chem. Phys. 2000, 112, 8910-8922. 
[31] C. Vega, J. L. F. Abascal, Phys. Chem. Chem. Phys. 2011, 13, 19663.

[32] C. Vega, J. L. F. Abascal, M. M. Conde, J. L. Aragones, Faraday Discuss. 2009, 141, 251276.

[33] W. A. Steele, Surf. Sci. 1973, 36, 317-352.

[34] S. J. Cox, S. M. Kathmann, J. A. Purton, M. J. Gillan, A. Michaelides, Phys. Chem. Chem. Phys. 2012, 14, 7944.

[35] W. A. Steele, Interaction of Gases at Solid Surfaces, Pergamon Press, Glasgow, 1974.

[36] A. Cheng, W. A. Steele, J. Chem. Phys. 1990, 92, 3858.

[37] A. Striolo, A. A. Chialvo, P. T. Cummings, K. E. Gubbins, Langmuir 2003, 19, 8583-8591.

[38] A. Striolo, K. E. Gubbins, A. A. Chialvo, P. T. Cummings, Mol. Phys. 2004, 102, 243-251.

[39] A. A. Chialvo, L. Vlcek, P. T. Cummings, J. Phys. Chem. C 2013, 117, 23875-23886.

[40] A. A. Chialvo, P. T. Cummings, J. Phys. Chem. A 2011, 115, 5918-5927.

[41] A. D. MacKerell, D. Bashford, J. Dunbrack Roland, L., J. D. Evanseck, M. J. Field, S. Fischer, J. Gao, H. Guo, S. Ha, D. Joseph-McCarthy, et al., J. Phys. Chem. B 1998, 102, 3586-3616.

[42] T. Werder, J. H. Walther, R. L. Jaffe, T. Halicioglu, P. Koumoutsakos, J. Phys. Chem. B 2003, $107,1345-1352$.

[43] B. Hess, C. Kutzner, D. Van Der Spoel, E. Lindahl, J. Chem. Theory Comput. 2008, 4, 435447.

[44] D. Van Der Spoel, E. Lindahl, B. Hess, G. Groenhof, A. E. Mark, H. J. C. Berendsen, J. Comput. Chem. 2005, 26, 1701-1718.

[45] D. van der Spoel, E. Lindhal, B. Hess, A. R. van Buuren, E. Apol, P. J. Meulenhoff, D. P. Tieleman, A. L. T. M. Sijbers, K. A. Feenstra, R. van Drunen, et al., Gromacs User Man. version 4.5.4 2010, www.gromacs.org.

[46] H. J. C. Berendsen, J. P. M. Postma, W. F. van Gunsteren, A. DiNola, J. R. Haak, J. Chem. Phys. 1984, 81, 3684-3690.

[47] R. W. Hockney, S. P. Goel, J. W. Eastwood, J. Comput. Phys. 1974, 14, 148-158.

[48] T. Darden, D. York, L. Pedersen, J. Chem. Phys. 1993, 98, 10089.

[49] U. Essmann, L. Perera, M. L. Berkowitz, T. Darden, H. Lee, L. G. Pedersen, J Chem Phys 1995, 103, 8577-8593.

[50] C. Avendaño, F. A. Escobedo, Soft Matter 2012, 8, 4675.

[51] D. R. Nelson, B. I. Halperin, Phys. Rev. B 1979, 19, 2457-2484.

[52] R. J. Gowers, M. Linke, J. Barnoud, T. J. E. Reddy, M. N. Melo, S. L. Seyler, D. L. Dotson, J. Domanski, S. Buchoux, I. M. Kenney, et al., in Proc. 15th Python Sci. Conf. (Ed.: S. Benthall and S. Rostrup), SciPy, Austin, TX, 2016, pp. 102-109.

[53] N. Michaud-Agrawal, E. J. Denning, T. B. Woolf, O. Beckstein, J. Comput. Chem. 2011, 32, 2319-2327. 
[54] P. Kumar, S. V. Buldyrev, F. W. Starr, N. Giovambattista, H. E. Stanley, Phys. Rev. E - Stat. Nonlinear, Soft Matter Phys. 2005, 72, 1-12.

[55] G. S. Kell, J. Chem. Eng. Data 1975, 20, 97-105.

[56] R. Zangi, A. E. Mark, J. Chem. Phys. 2003, 119, 1694-1700.

[57] V. Petrenko, R. Whitworth, Physics of Ice, Oxford University Press, Oxford, 1999.

[58] J. L. F. Abascal, C. Vega, Phys. Rev. Lett. 2007, 98, 2005-2008.

[59] R. Kumar, J. R. Schmidt, J. L. Skinner, J. Chem. Phys. 2007, 126, 204107.

[60] B. Chen, J. Ilja Siepmann, J. Phys. Chem. B 2006, 110, 3555-3563.

[61] O. Gereben, L. Pusztai, J. Phys. Chem. B 2015, 119, 3070-3084.

[62] D. Prada-Gracia, R. Shevchuk, F. Rao, J. Chem. Phys. 2013, 139, 84501.

[63] A. M. Zaki, A. Troisi, P. Carbone, J. Phys. Chem. Lett. 2016, 7, 3730-3735.

[64] R. J. Gowers, P. Carbone, J. Chem. Phys. 2015, 142, 0-10.

[65] J. Zielkiewicz, J. Chem. Phys. 2005, 123, 0-6. 